\title{
The Effects of Green Kiwifruit Ingestion on Digestive Health, Blood Flow, Skin Health, and the Autonomic Nervous System
}

\author{
Fumi Ishida ${ }^{1}$, Ailing Hu${ }^{2}$, Takuji Yamaguchi², Yuna Naraoka1, Hiroyuki Kobayashi', ${ }^{1}$ \\ ${ }^{1}$ Department of Hospital Administration, Graduate School of Medicine, Juntendo University, Tokyo, Japan \\ ${ }^{2}$ Center for Advanced Kampo Medicine and Clinical Research, Graduate School of Medicine, Juntendo University, Tokyo, Japan \\ Email: f-ishida@juntendo.ac.jp
}

How to cite this paper: Ishida, F., Hu, A.L., Yamaguchi, T., Naraoka, Y. and Kobayashi, H. (2021) The Effects of Green Kiwifruit Ingestion on Digestive Health, Blood Flow, Skin Health, and the Autonomic Nervous System. Health, 13, 647-659. https://doi.org/10.4236/health.2021.136049

Received: April 26, 2021

Accepted: June 6, 2021

Published: June 9, 2021

Copyright ( 2021 by author(s) and Scientific Research Publishing Inc. This work is licensed under the Creative Commons Attribution International License (CC BY 4.0).

http://creativecommons.org/licenses/by/4.0/ (c) (i) Open Access

\begin{abstract}
We aimed to assess the impact of consuming two Zespri Hayward green kiwifruit a day on 11 healthy women subjects over a period of 4 consecutive weeks. We analyzed the effects of kiwifruit consumption on intestinal, abdominal, and bowel movement related discomfort, bowel movement frequency, peripheral blood flow, skin health, and autonomic nervous system activity. This study was a randomized control trial, consisting of a four-week intervention period. Participants were divided to ensure equal age distributions and then randomly placed into two intervention groups: a kiwifruit consumption group ( $\mathrm{n}=11)$ and a control group (no kiwifruit consumption) ( $\mathrm{n}$ $=5$ ). The kiwifruit consumption group was instructed to consume two kiwifruit a day during the 4-week intervention period, while subjects in the control group were instructed not to consume kiwifruit for the duration of the study. Besides a restriction from eating yogurt and other fruits, subjects were requested to eat their meals as per usual. The results of this study showed a significant decrease in intestinal, abdominal, and bowel movement discomfort, and a significant increase in bowel movement frequency, at 4 weeks of kiwifruit consumption compared to before consumption. In addition, subjects in the kiwifruit consumption group experienced a significant increase in blood flow, particularly in the index and middle finger at 2 and 4 weeks of kiwifruit consumption. Furthermore, subjects in the kiwifruit consumption group experienced a significant increase in skin brightness at 4 weeks of kiwifruit consumption compared to before consumption. The results of this study suggest that kiwifruit appears to be a delicious and safe option for intestinal, abdominal, and bowel movement discomfort interventions for healthy women. In addition, kiwifruit may aid in increasing bowel movement frequency, peripheral blood flow, and brightness of skin among healthy women as well.
\end{abstract}




\section{Keywords}

Kiwifruit, Dietary Fiber, Digestion, Autonomic Nervous System, Blood Flow, Skin Health

\section{Introduction}

Kiwifruit is one of the most reputable superfoods, known for its remarkable health benefits, particularly for digestive health. Kiwifruit originated in the temperate forests of southwest China [1]. In the early 1900's, kiwifruit seeds were brought to New Zealand, and since then, kiwifruit has been grown and commonly consumed across the world [2]. The two main varieties of kiwifruit are known as Actinidia deliciosa "Hayward," more widely known as green kiwifruit, and Actinidia chinensis, more commonly known as gold kiwifruit [1] [2]. Although green and gold kiwifruit differ in color, texture, and taste, they both contain exceptionally high levels of a variety of nutrients and antioxidants, most notably vitamin $\mathrm{C}$, dietary fiber, potassium, vitamin $\mathrm{E}$, and folate [1]. The kiwifruit has been reported as the most nutrient dense among commonly eaten fruits [3], meaning that it is high in nutrients and low in calories. These beneficial health properties have made kiwifruit an exciting subject of interest for potential therapeutic interventions.

One particular growing area of research is the relationship between kiwifruit and gut health, with many studies supporting the notion that kiwifruit can improve gut health by "promoting gastrointestinal motility, facilitating digestion, and inducing laxation" [4]. A clinical study conducted by Rush et al., was one of the first controlled human studies to investigate the laxative effects of kiwifruit on people above the age of 60 , who often experience constipation and impaired bowel function. The results from this study confirmed the anecdotal assumption that kiwifruit contains laxative properties, and found that kiwifruit produces a looser or softer stool and greater bulk [5].

Two unrelated studies also investigated the laxative properties of kiwifruit on patients in two separate regions of China. One study included patients suffering from constipation [6] and the other study included patients with constipationpredominant irritable bowel syndrome (IBS/C) [7]. The first study used a 4-week treatment period where 33 constipated patients and 22 healthy subjects ate 2 Zespri kiwifruit a day. A significant increase in complete spontaneous bowel motion (CSBM), improved transit time and rectal sensation, and decreased reported use of laxatives was found in the constipation group. In addition, although there was a change in anorectal physiology in constipated patients, no significant change was detected in the healthy control subjects [6]. The second study separated IBS/C patients into two groups: an experimental treatment group and a placebo group. In total, 60 IBS/C patients participated, along with 16 additional healthy subjects, who served as a positive control group. 45 IBS/C patients 
and 16 healthy subjects consumed two Hayward green kiwifruits a day over a span of 4 weeks, while 15 IBS/C patients in the placebo group ingested two placebo capsules of 0.75 grams of glucose powder a day for 4 weeks. The IBS/C patients in the experimental group experienced a significant increase in bowel movement frequency, decreased transit time, and improved bowel function after 4 weeks of kiwifruit ingestion. No significant changes in fecal volume, bowel movement frequency, and transit time were reported throughout the intervention period in the healthy subjects. Furthermore, significant increases in bowel movement were observed only in the treatment group, suggesting that kiwifruit may be a safe and effective natural laxative for patients diagnosed with IBS/C [7]. This is consistent with results from previous studies that suggest that kiwifruit consumption can benefit adults and elders with constipation. Although both studies focused on different sample groups and outcome measures, each study reported positive results in terms of gut health, suggesting that kiwifruit may be a promising intervention for those with constipation and those with IBS/C [7].

Although these studies focused on different target populations, all of these studies linked kiwifruit's unique dietary fiber composition, in addition to its abundance of phytochemicals, to its enhanced laxative properties. Kiwifruit contains approximately $2 \%$ to $3 \%$ dietary fiber [2] and, according to a report in April 2018 by the US Department of Agriculture, green kiwifruit contains about 3 grams of fiber per 100 grams of edible kiwifruit [8]. In Japan, the recommended daily fiber intake for adults is at least 18 and 20 grams for women and men respectively [9], meaning that kiwifruit provides over $10 \%$ of the recommended daily requirement for dietary fiber. Kiwifruit contains both soluble and insoluble dietary fibers [10]. Both of these fibers have mechanisms that create an increase in water content, resulting in soft, bulky stools, ultimately driving a laxative effect [11]. Dietary fiber also influences stool and stool consistency, with different types of fibers encompassing unique mechanisms which promote the increase of stool bulk, decrease in transit time, as well as other gut-related benefits [12]. Additionally, kiwifruit fibers have unique hydration properties, such as "water retention, capacity and swelling, and viscosity" [1]. The cell walls of ripe kiwifruit have the potential to swell up to 3 to 4 times the size of the cell walls of an unripe kiwifruit [7] [13]. This ability illustrates the high water-holding capacity of the dietary fiber found in kiwifruit [7]. Another factor that is suspected to contribute to these laxative properties in green kiwifruit is actinidin, a proteolytic enzyme which has been linked to reducing transit time in the GI tract [14] [15] and aid in protein digestion [16].

In addition to having a high amount of dietary fiber, kiwifruit also contains high quantities of vitamin $\mathrm{C}, \mathrm{E}$, and potassium. The USDA reports that green kiwifruit contains approximately $92.7 \mathrm{mg}$ of vitamin C per 100 grams of edible kiwifruit [8]. Vitamin C is associated with mood regulation [17] [18] and collagen production [4]. The vitamin $\mathrm{E}$ in kiwifruit may contribute to widening 
blood vessels and aiding in the prevention of blood clots [19] [20]. Furthermore, there are $312 \mathrm{mg}$ of potassium per 100 grams of edible green kiwifruit [8]. Potassium helps regulate muscle and heart contractions [21], and therefore kiwifruit may have a positive influence on autonomic nervous system activity.

Although the unique properties of dietary fiber, actinidin, vitamin C, vitamin $\mathrm{E}$, and potassium found in green kiwifruit have all been investigated separately, there have been very few studies investigating the comprehensive effects of these nutrients on healthy subjects. We hypothesize that the cumulative effect of these nutrients creates a multi-directional series of health benefits between the gut, brain (gut-brain axis), and epidermis. We aimed to assess the impact of consuming two Zespri Hayward green kiwifruit a day on 11 healthy women subjects over a period of 4 consecutive weeks. We analyzed the effects of kiwifruit consumption on intestinal, abdominal, and bowel movement related discomfort, bowel movement frequency, peripheral blood flow, skin health, and autonomic nervous system activity.

\section{Materials and Methods}

\subsection{Participants}

A total of 16 women were recruited through study advertisements placed at Juntendo University in Tokyo, Japan. The specific inclusion criteria were as follows: healthy women aged 20 - 59 years old. Exclusion criteria were as follows: persons who use external medications that may influence study results or those deemed unsuitable for participation per the discretion of a medical doctor. After a pre-study screening, 16 total eligible women were selected for participation. The average participant age was $31.5 \pm 1.3$ years [mean \pm standard error (SE)]. All of the subjects were informed about the possible risks and discomforts that may occur during the experiment prior to receiving their written informed consent to voluntarily participate in the study. This study's design was approved by the Juntendo University Ethical Review Board. Furthermore, all procedures were conducted in accordance with the principles of the amended Declaration of Helsinki.

\subsection{Test Food Product}

The kiwifruit used in this experiment was provided by Zespri International (Japan) K.K., Tokyo, Japan. Each kiwifruit contained about 3 grams of fiber per 100 grams. Because each participant ate two kiwifruit a day, they ingested about 6 grams of fiber per day.

\subsection{Procedures}

This study was a randomized controlled trial consisting of a four-week intervention period. Participants were divided to ensure equal age distribution and then randomly placed into two intervention groups: a kiwifruit consumption group $(\mathrm{n}=11)$ and a control group (no kiwifruit consumption) $(\mathrm{n}=5)$. The kiwifruit 
consumption group was instructed to consume two kiwifruit a day during the 4-week intervention period, while subjects in the control group were instructed not to consume kiwifruit for the duration of the study. Besides a restriction from eating yogurt and other fruits, subjects in both groups were requested to eat their meals per usual. All assessments were measured at Juntendo University before the start of the study, and at two-week and four-week intervals, after applying for the study. This study was conducted from July 2018 to May 2019 at Juntendo University.

\subsection{Bowel Movement Assessment}

The effect of kiwifruit consumption on bowel movement was evaluated by the Japanese version of the Constipation Assessment Scale (CAS). Interviews were also conducted to gather further information, such as how many bowel movements subjects had in a day. Data before kiwifruit consumption, at two weeks of kiwifruit consumption, and at 4 weeks of kiwifruit consumption were collected and compared.

\subsection{Skin Blood Flow Assessment}

Subjects rested in a $26^{\circ} \mathrm{C}-28^{\circ} \mathrm{C}$ room for 30 minutes before skin blood flow assessment procedures began, in order to give subjects ample time to adjust to the conditions of the room. Blood flow of the index finger, middle finger, and ring finger was measured with a laser speckle blood flow imager, MoorFLPI (zero c seven, Inc., Tokyo Japan), for an average of one minute each. We designated the amount of blood flow before kiwifruit consumption to be at a baseline of 100, and recorded an increase or decrease of blood flow at 2 weeks and 4 weeks of kiwifruit consumption compared to baseline.

\subsection{Skin Condition Assessment}

Skin condition analysis was taken 30 minutes after the subjects washed their face. RoboSkin Analyzer (MM Neek) was used to analyze skin tone, age spots, and skin texture. The amount of moisture in the subject's skin was measured using SKIN DIAGNOSTIC (Courage + Khazaka, Cologne, Germany). We designated the amount of moisture in the skin before kiwifruit consumption to be at 100 , and recorded an increase or decrease in moisture at 2 weeks and 4 weeks of kiwifruit consumption.

\subsection{Autonomic Nervous System Activity Assessment}

An autonomic nervous system analysis acceleration pulse wave meter, (Pulse Analyzer Plus TAS9, YKC group), was used to measure autonomic nervous system activity. We attached the sensor to the index (second) finger of the left hand, and measured autonomic nervous system activity for 5 minutes. Data concerning the amount and balance of autonomic nervous system activity before kiwifruit consumption, at two weeks of kiwifruit consumption, and at 4 weeks of kiwifruit consumption were collected and compared. 


\subsection{Statistical Analysis}

This study conducted statistical tests using SPSS ver. 26.0 (SPSS, IBM) (all significance levels were set to $\mathrm{p}<0.05)$. All measurement items were assessed using the paired $t$-test. All data is expressed as means \pm standard errors, unless otherwise specified.

\section{Results}

\subsection{Bowel Movement Assessment}

Compared to the control group, the kiwifruit consumption group displayed a significant decrease in intestinal, abdominal, and bowel movement related discomfort at 4 weeks $(\mathrm{p}<0.05)$ compared to week 0. (Figure 1(a)). Furthermore, the number of defecations per week also increased significantly in the kiwifruit consumption group at week 4 compared to week 0. $(\mathrm{p}<0.05)$ (Figure $1(\mathrm{~b})$ )

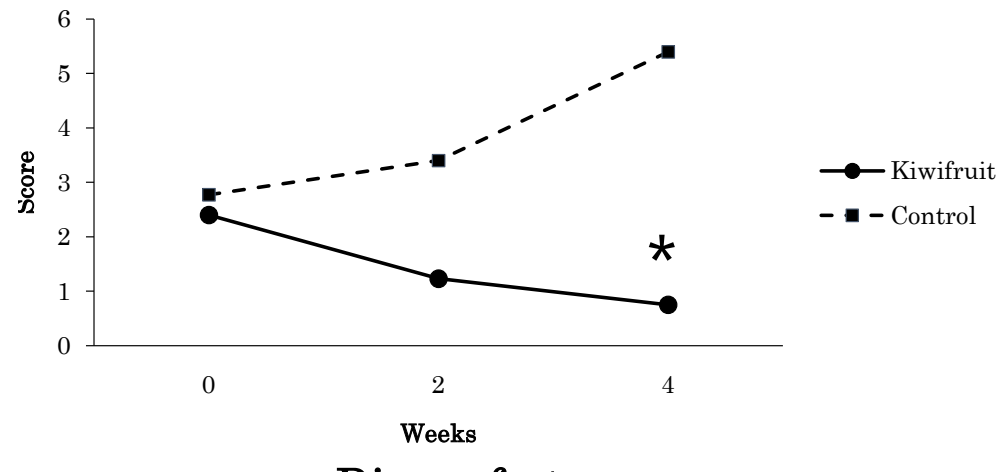

(a)

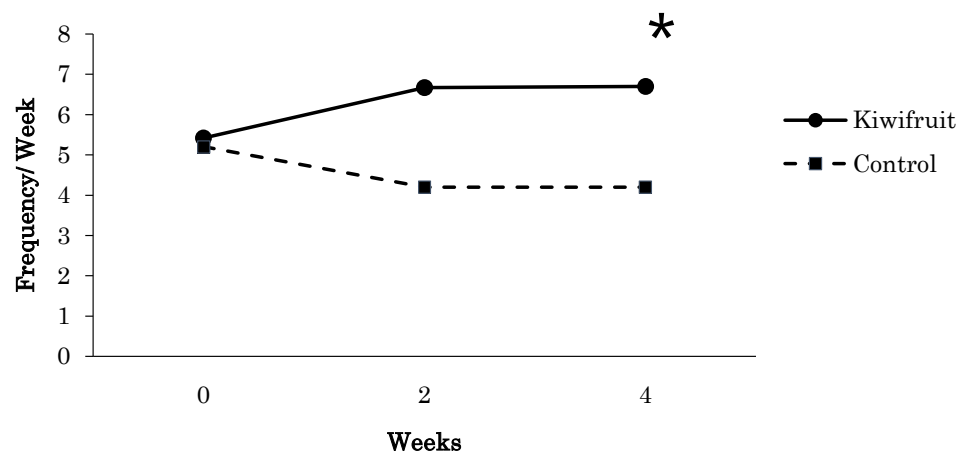

Stool Frequency

(b)

Figure 1. (a) Stool frequency in the kiwifruit consumption group $(n=11)$ and control group $(n=5)$. Discomfort was measured using the Japanese version of CAS. The score values are based on the following scale: 1) feeling of complete evacuation, 2) feeling of moderate evacuation, 3) feeling of slight evacuation, 4) not sure, 5) slight feeling of discomfort, 6) feelings of discomfort. (b) Stool frequency per week was disclosed during an interview session with the subject. Results are expressed as mean \pm S.E. ${ }^{*} \mathrm{p}<0.05$ vs. control. 


\subsection{Skin Blood Flow Assessment}

Subjects in the kiwifruit consumption group displayed a significant improvement in blood flow of the index finger at 2 weeks $(p=0.024)$ and 4 weeks $(p=$ 0.029) compared to before kiwifruit consumption. Subjects in the kiwifruit consumption group also displayed a significant improvement in blood flow of the middle finger at 2 weeks $(p=0.031)$ and 4 weeks $(p=0.027)$ compared to before kiwifruit consumption (Figure 2).

\subsection{Skin Condition Assessment}

Subjects in the kiwifruit consumption group experienced a significant increase in skin brightness at 4 weeks of consumption compared to before consumption $(\mathrm{p}=0.037)$ (Data not shown). Subjects in the control group experienced a significant decrease in skin brightness at 4 weeks compared to 0 weeks. (Data not shown).

\subsection{Autonomic Nervous System Activity Assessment}

No significant changes in autonomic nervous system activity was found in either group (Data not shown).

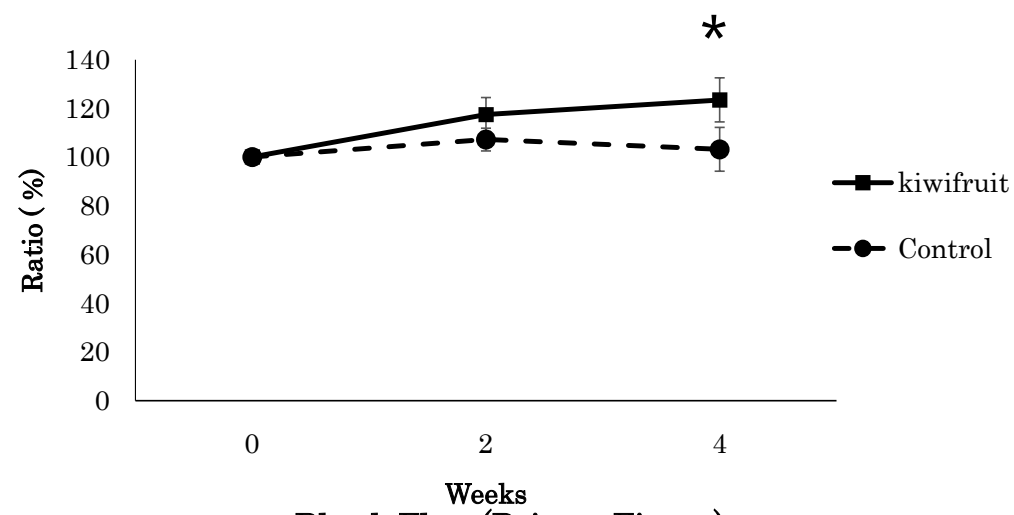

Blood Flow (Pointer Finger)

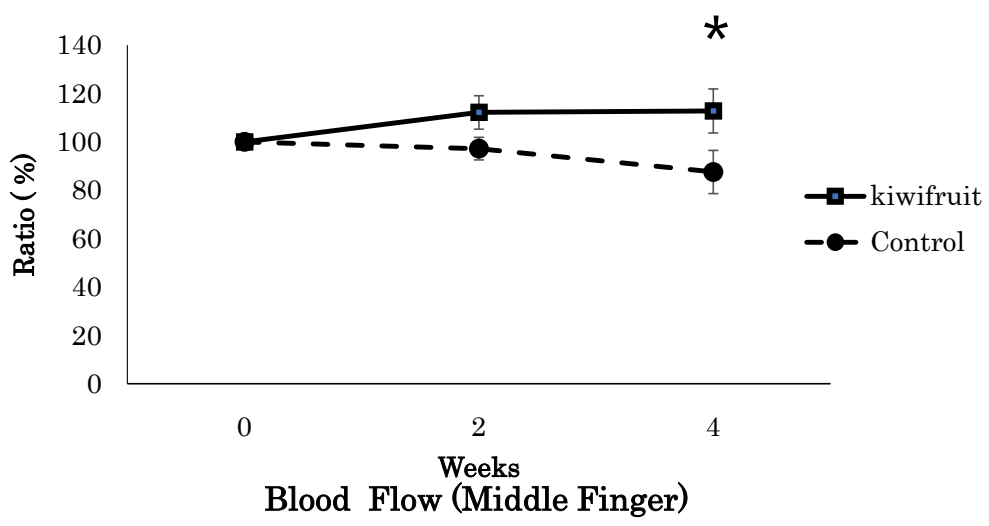

Figure 2. Comparison of blood flow between the kiwifruit consumption group $(n=11)$ and the control group $(n=5)$. Results are expressed as mean \pm S.E. ${ }^{*} \mathrm{p}<0.05$ vs. control. 


\section{Discussion}

In this current study, we examined the effects of eating two Zespri Hayward green kiwifruit a day for 4 weeks on 11 healthy women subjects and compared the results to a control group in which individuals were instructed to refrain from eating kiwifruit during the intervention period. Our study looked at the effect of kiwifruit consumption on intestinal, abdominal, and bowel movement related discomfort, bowel frequency, peripheral blood flow, skin health, and autonomic nervous system activity. We found that individuals in the kiwifruit consumption group experienced a significant decrease in intestinal, abdominal, and bowel movement related discomfort and a significant increase in the number of defecations per week at 4 weeks of consumption compared to before consumption. In addition, we also found a significant increase in the amount of blood flow, specifically in the index and middle finger, at 2 weeks and 4 weeks compared to before consumption. Furthermore, a significant increase in skin brightness was found in the kiwifruit consumption group, whereas a significant decrease in skin brightness was found in the control group. Although a significant change in autonomic nervous system activity was not detected in this study, it is possible that the autonomic nervous system played a role in the significant increase in blood flow and skin brightness seen in the kiwifruit consumption group, due to the increase in dietary fiber intake by these subjects. Further studies are needed to investigate this correlation.

The results of this study showed that the subjects in the kiwifruit consumption group experienced a significant decrease in intestinal, abdominal, and bowel movement related discomfort at 4 weeks of kiwifruit consumption compared to before consumption. Furthermore, the number of bowel movements per week also significantly increased in the kiwifruit consumption group at 4 weeks of consumption in comparison to before. This is consistent with numerous studies that suggest kiwifruit can improve gut health. A study conducted by Sarah Eady et al., set out to determine if daily consumption of three gold-fleshed kiwifruit could improve gastrointestinal discomfort and relieve constipation in subjects with mild constipation. This study found that subjects in the kiwifruit consumption group experienced a significantly greater number of complete spontaneous bowel movements (CSBM) a week, in addition to improved gastrointestinal discomfort, compared with baseline for abdominal pain, constipation, and indigestion [22]. Another recent study conducted by Noemi Caballero et al., investigated the effect of green kiwifruit on transit and tolerance to intestinal gas in 11 healthy subjects [23]. This randomized, cross-over study found that a diet consisting of 2 weeks of daily consumption of kiwifruit, in addition to a low-flatulogenic diet, led to an increase in stool frequency compared to a diet without kiwifruit for 2 weeks. This study also found that although stool frequency increased in the kiwifruit consumption group, subjects did not undergo associated abdominal symptoms, such as increased rectal gas evacuation, which can occur due to an increase in fiber intake. This study concluded that green kiwifruit can in- 
crease stool frequency in healthy subjects without the associated effects on intestinal gas transit and tolerance, making it a potential alternative treatment for constipation [23]. In addition to laxative properties, Lee et al. wanted to determine how 4 days of kiwifruit consumption would influence intestinal microbiota populations [24]. Although the observed effects were short-term and different for each individual, this study observed an increase in the intestinal lactic acid bacteria, lactobacilli, in four out of six healthy Chinese female adults within 24 hours of ingesting the equivalent of two kiwifruit in the form of freeze-dried kiwifruit powder. In addition, five out of the six subjects experienced a significant increase of intestinal bifidobacteria, compared to their baseline data, after 4 days of kiwifruit consumption. Furthermore, although not significant, an apparent change in the population of Clostridium and Bacteriodes was observed during kiwifruit ingestion. These results suggest that kiwifruit may have selective prebiotic effects on the intestinal microbiota [24].

In addition to gut health benefits, the present study also revealed the potential role kiwifruit may play in increasing peripheral blood flow in healthy women. The results displayed a significant increase in blood flow in the index fingers of participants from the kiwifruit consumption group, demonstrating a $123.5 \%$ increase in peripheral blood flow at week 4 compared to week 0 . A significant increase in blood flow was also found in the middle fingers of subjects from the kiwifruit consumption group as well. This finding is consistent with studies investigating the relationship between kiwifruit consumption and blood pressure. A study published in the Journal of Human Hypertension by Karlsen et al. was one of the first studies to investigate the effects of kiwifruit on blood pressure. This study demonstrated that ingesting 3 kiwifruits daily, over 8 weeks' time, promotes "pronounced anti-hypertensive and anti-thrombotic effect(s) in male smokers" [25], suggesting a potential role kiwifruit may play in improving peripheral blood flow. This effect on blood flow may be attributed to the large amount of vitamin $\mathrm{E}$ found in kiwifruit. Vitamin $\mathrm{E}$ aids in widening blood vessels and preventing blood clots [19] [20].

In addition, skin protective properties of kiwifruit were also demonstrated throughout the study. Through the use of RoboSkin Analyzer, we were able to measure overall skin tone, age spots, and skin texture. SKIN DIAGNOSTIC was also used to measure the amount of moisture in the subject's skin. While individuals in the control group experienced a significant decrease in skin brightness, participants in the kiwifruit consumption group experienced a significant increase in skin brightness at 4 weeks of consumption compared to before consumption. According to the USDA, green kiwifruit contains approximately 92.7 $\mathrm{mg}$ of vitamin C per 100 grams of edible kiwifruit [8]. Because vitamin $\mathrm{C}$ is associated with collagen production [4], the increased vitamin C consumption in the kiwifruit consumption group may have contributed to the significant improvement of skin brightness in these subjects.

In this study, we were unable to detect a significant change in autonomic 
nervous system activity in subjects from both the kiwifruit consumption group and the control group. Although a significant change in autonomic nervous system activity was not found, it may have played a role in mediating the significant increase in bowel frequency, peripheral blood flow, and skin brightness in subjects in the kiwifruit consumption group due to the increase in dietary fiber intake. To our knowledge, there have not been any other studies investigating the relationship between kiwifruit ingestion and autonomic nervous system activity. That being said, recent studies have revealed the importance of the autonomic nervous system in relation to gut health, including regulating motility, secretion, and vasoregulation of the gastrointestinal tract [26]. About 30 years ago, it was discovered that those who suffer from IBD also suffer from autonomic dysfunction, demonstrating a link between the autonomic nervous system and gut functionality [26] [27]. A separate study investigated the relationship between the lack of dietary fiber consumption on microbiota composition and physiology in addition to the resulting effects on the mucus barrier of the colon using a gnotobiotic mouse model, colonized with a synthetic human gut microbiota [28]. This study found that depriving gut microbiota of dietary fiber results in the erosion of the intestinal mucus barrier, promoting increased susceptibility to pathogens. The results of this study suggest that diet, in particular dietary fiber, plays a vital role in regulating gut health [28]. Although further investigation into the bi-directional relationship between the gut and the brain is still needed, many studies support the hypothesis that improving gut health through dietary measures, such as increasing dietary fiber, can lead to better gut health and autonomic nervous system activity. Specific studies further investigating the relationship between the fiber-filled kiwifruit and the autonomic nervous system are still needed.

Due to the small sample size of this study, further studies with a larger sample size should be conducted. In addition, a longer intervention period may provide further insight into the long-term health benefits of eating 2 green kiwifruits a day. Furthermore, while this study consisted of healthy women participants, it may be interesting to conduct the same experiment on different groups of individuals, particularly those suffering from gastrointestinal dysfunctions, such as constipation or IBS.

Research investigating the potential health benefits of kiwifruit has become a growing interest amongst researchers and physicians alike within the past decade, due to the promising results of numerous studies which suggest kiwifruit may provide a variety of health benefits due to their unique, nutrient-dense properties. The results of this study suggest that kiwifruit appears to be a delicious and safe intervention option for intestinal, abdominal, and bowel movement related discomfort. In addition, kiwifruit may help increase bowel frequency and peripheral blood flow, as well as improve skin brightness in healthy women. Due to the kiwifruit's unique nutrient-dense and fiber-filled composition, it is possible that a number of factors may be associated with the positive health benefits found in this study, and that these benefits could potentially be mediated through a crosstalk between the brain, the skin, and the gastrointestinal environment. 
This study provides a solid baseline for future studies to further explore this relationship between kiwifruit consumption, the microbiome, skin health, and the autonomic nervous system, among a larger, and more diverse group of subjects, for longer intervention periods.

\section{Conclusion}

In conclusion, we found that consuming two Zespri Hayward green kiwifruit a day for 4 weeks may aid in alleviating bowel movement related discomfort, increase stool frequency and peripheral blood flow, and aid in improving skin brightness among healthy women.

\section{Acknowledgements}

The authors would like to thank all the women and professional staff who participated in this study.

\section{Conflicts of Interest}

The authors declare no conflicts of interest regarding the publication of this paper.

\section{References}

[1] Richardson, D.P., Ansell, J. and Drummond, L.N. (2018) The Nutritional and Health Attributes of Kiwifruit: A Review. European Journal of Nutrition, 57, 2659-2676. https://doi.org/10.1007/s00394-018-1627-Z

[2] Singletary, K. (2012) Kiwifruit: Overview of Potential Health Benefits. Nutrition Today, 47, 133-147. https://doi.org/10.1097/NT.0b013e31825744bc

[3] Stonehouse, W., Gammon, C.S., Beck, K.L., Conlon, C.A., von Hurst, P.R. and Kruger, R. (2013) Kiwifruit: Our Daily Prescription for Health. Canadian Journal of Physiology and Pharmacology, 91, 442-447. https://doi.org/10.1139/cjpp-2012-0303

[4] Cundra, L.B., D’Souza, S.M., Parekh, P.J. and Johnson, D.A. (2020) The Impact of Kiwifruit on Human Health. Gut Gastroenterology, 3, 1-10.

[5] Rush, E.C., Patel, M., Plank, L.D. and Ferguson, L.R. (2002) Kiwifruit Promotes Laxation in the Elderly. Asia Pacific Journal of Clinical Nutrition, 11, 164-168. https://doi.org/10.1046/j.1440-6047.2002.00287.x

[6] Chan, A.O.O., Leung, G., Tong, T. and Wong, N.Y. (2007) Increasing Dietary Fiber Intake in Terms of Kiwifruit Improves Constipation in Chinese Patients. World Journal of Gastroenterology, 13, 4771-4775. https://doi.org/10.3748/wjg.v13.i35.4771

[7] Chang, C.C., Lin, Y.T., Lu, Y.T., Liu, Y.S. and Liu, J.F. (2010) Kiwifruit Improves Bowel Function in Patients with Irritable Bowel Syndrome with Constipation. Asia Pacific Journal of Clinical Nutrition, 19, 451.

[8] United States Department of Agriculture (2018) Green and Gold Kiwifruit. USDA National Nutrient Database for Standard Reference, Release 28. Version: April 2018. US Department of Agriculture (USDA), Agricultural Research Service (ARS), Nutrient Data Laboratory, Beltsville (MD). https://fdc.nal.usda.gov/fdc-app.html\#/food-details/168153/nutrients

[9] Ministry of Health, Labour and Welfare, Japan (2015) Overview of Dietary Reference Intakes for Japanese. Ministry of Health, Labour and Welfare, Japan, Tokyo. 
https://www.mhlw.go.jp/file/06-Seisakujouhou-10900000-Kenkoukyoku/Overview. pdf

[10] Carnachan, S.M., Bootten, T.J., Mishra, S., Monro, J.A. and Sims, I.M. (2012) Effects of Simulated Digestion in Vitro on Cell Wall Polysaccharides from Kiwifruit (Actinidia spp.). Food Chemistry, 133, 132-139.

https://doi.org/10.1016/j.foodchem.2011.12.084

[11] McRorie Jr., J.W. and McKeown, N.M. (2017) Understanding the Physics of Functional Fibers in the Gastrointestinal Tract: An Evidence-Based Approach to Resolving Enduring Misconceptions about Insoluble and Soluble Fiber. Journal of the Academy of Nutrition and Dietetics, 117, 251-264. https://doi.org/10.1016/j.jand.2016.09.021

[12] Dhingra, D., Michael, M., Rajput, H. and Patil, R.T. (2012) Dietary Fibre in Foods: A Review. Journal of Food Science and Technology, 49, 255-266. https://doi.org/10.1007/s13197-011-0365-5

[13] Hallett, I.C., Macrae, E.A. and Wegrzyn, T.F. (1992) Changes in Kiwifruit Cell Wall Ultrastructure and Cell Packing during Postharvest Ripening. International Journal of Plant Sciences, 153, 49-60. https://doi.org/10.1086/297006

[14] Bayer, S.B., Gearry, R.B. and Drummond, L.N. (2018) Putative Mechanisms of Kiwifruit on Maintenance of Normal Gastrointestinal Function. Critical Reviews in Food Science and Nutrition, 58, 2432-2452. https://doi.org/10.1080/10408398.2017.1327841

[15] Montoya, C.A., Rutherfurd, S.M., Olson, T.D., Purba, A.S., Drummond, L.N., Boland, M.J. and Moughan, P.J. (2014) Actinidin from Kiwifruit (Actinidia deliciosa cv. Hayward) Increases the Digestion and Rate of Gastric Emptying of Meat Proteins in the Growing Pig. British Journal of Nutrition, 111, 957-967. https://doi.org/10.1017/S0007114513003401

[16] Kaur, L., Rutherfurd, S.M., Moughan, P.J., Drummond, L. and Boland, M.J. (2010) Actinidin Enhances Gastric Protein Digestion as Assessed Using an in Vitro Gastric Digestion Model. Journal of Agricultural and Food Chemistry, 58, 5068-5073. https://doi.org/10.1021/jf903332a

[17] Gupta, P., Tiwari, S. and Haria, J. (2014) Relationship between Depression and Vitamin C Status: A Study on Rural Patients from Western Uttar Pradesh in India. International Journal of Scientific Study, 1, 37-39.

[18] Naidu, K.A. (2003) Vitamin C in Human Health and Disease Is Still a Mystery? An Overview. Nutrition Journal, 2, Article No. 7. https://doi.org/10.1186/1475-2891-2-7

[19] National Institute of Mental Health (2021) Vitamin E [Fact Sheet for Consumers]. https://ods.od.nih.gov/factsheets/VitaminE-Consumer/

[20] Wennmalm, P.A. (1994) Endothelial Nitric Oxide and Cardiovascular Disease. Journal of Internal Medicine, 235, 317-327. https://doi.org/10.1111/j.1365-2796.1994.tb01081.x

[21] Sonawane, M.S. (2018) Exploring Nutritional Values, Health Claims and Allergies Associated with Kiwifruit Consumption. Plant Archives, 18, 2545-2552.

[22] Eady, S.L., Wallace, A. J., Butts, C.A., Hedderley, D., Drummond, L., Ansell, J. and Gearry, R.B. (2019) The Effect of "Zesy002" Kiwifruit (Actinidia chinensis var. chinensis) on Gut Health Function: A Randomised Cross-Over Clinical Trial. Journal of Nutritional Science, 8, e18. https://doi.org/10.1017/jns.2019.14

[23] Caballero, N., Benslaiman, B., Ansell, J. and Serra, J. (2020) The Effect of Green Kiwifruit on Gas Transit and Tolerance in Healthy Humans. Neurogastroenterology \& 
Motility, 32, e13874. https://doi.org/10.1111/nmo.13874

[24] Kun Lee, Y., Yi Low, K., Siah, K., Drummond, L.M. and Gwee, K.A. (2012) Kiwifruit (Actinidia deliciosa) Changes Intestinal Microbial Profile. Microbial Ecology in Health and Disease, 23, 18572. https://doi.org/10.3402/mehd.v23i0.18572

[25] Karlsen, A., Svendsen, M., Seljeflot, I., Laake, P., Duttaroy, A.K., Drevon, C.A., et al. (2013) Kiwifruit Decreases Blood Pressure and Whole-Blood Platelet Aggregation in Male Smokers. Journal of Human Hypertension, 27, 126-130. https://doi.org/10.1038/jhh.2011.116

[26] Brinkman, D.J., Ten Hove, A.S., Vervoordeldonk, M.J., Luyer, M.D. and de Jonge, W.J. (2019) Neuroimmune Interactions in the Gut and Their Significance for Intestinal Immunity. Cells, 8, 670. https://doi.org/10.3390/cells8070670

[27] Lindgren, S., Stewenius, J., Sjölund, K., Lilja, B. and Sundkvist, G. (1993) Autonomic Vagal Nerve Dysfunction in Patients with Ulcerative Colitis. Scandinavian Journal of Gastroenterology, 28, 638-642. https://doi.org/10.3109/00365529309096103

[28] Desai, M.S., Seekatz, A.M., Koropatkin, N.M., Kamada, N., Hickey, C.A., Wolter, M., et al. (2016) A Dietary Fiber-Deprived Gut Microbiota Degrades the Colonic Mucus Barrier and Enhances Pathogen Susceptibility. Cell, 167, 1339-1353.

https://doi.org/10.1016/j.cell.2016.10.043 